\title{
Boundary Control Problem of Infinite Order Distributed Hyperbolic Systems Involving Time Lags
}

\author{
Bahaa Gaber Mohamed \\ Department of Mathematics, Faculty of Science, Beni-Suef University, Beni-Suef, Egypt \\ Email: Bahaa_gm@hotmail.com
}

Received December 5, 2010; revised May 16, 2012; accepted May 23, 2012

\begin{abstract}
Various optimal boundary control problems for linear infinite order distributed hyperbolic systems involving constant time lags are considered. Constraints on controls are imposed. Necessary and sufficient optimality conditions for the Neumann problem with the quadratic performance functional are derived.
\end{abstract}

Keywords: Boundary Control; $(n \times n)$ Hyperbolic Systems; Time Lags; Distributed Control Problems; Neumann Conditions; Existence and Uniqueness of Solutions; Infinite Order Operator

\section{Introduction}

Distributed parameters systems with delays can be used to describe many phenomena in the real world. As is well known, heat conduction, properties of elastic-plastic material, fluid dynamics, diffusion-reaction processes, the transmission of the signals at a certain distance by using electric long lines, etc., all lie within this area. The object that we are studying (temperature, displacement, concentration, velocity, etc.) is usually referred to as the state.

The optimal control problems of second order distributed parabolic and hyperbolic systems involving time lags appearing in the boundary condition have been widely discussed in many papers and monographs. A fundamental study of such problems is given by [1] and was next developed by [2] and [3]. It was also intensively investigated by [4-14] and [15,16] in which linear quadratic problem for parabolic and hyperbolic systems with time delays given in the different form (constant time delays, time-varying delays, time delays given in the integral form, etc.) were presented.

In this paper, we consider the optimal control for infinite order hyperbolic systems and for $(n \times n)$ infinite order hyperbolic systems involving constant time lags appearing in both in the state equation and in the boundary condition. Such an infinite order hyperbolic system can be treated as a generalization of the mathematical model for a plasma control process.

The quadratic performance functional defined over a fixed time horizon are taken and some constraints are imposed on the boundary control. Following a line of the Lions scheme, necessary and sufficient optimality conditions for the Neumann problem applied to the above system were derived. The optimal control is characterized by the adjoint equations.

This paper is organized as follows. In Section 1, we introduce spaces of functions of infinite order. In Section 2, we formulate the mixed Neumann problem for infinite order hyperbolic systems involving constant time lags. In Section 3 , the boundary optimal control problem for this case is formulated, then we give the necessary and sufficient conditions for the control to be an optimal. In Section 4, we concluded and generalized our results.

\section{Sobolev Spaces with Infinite Order}

The object of this section is to give the definition of some function spaces of infinite order, and the chains of the constructed spaces which will be used later.

Let $\Omega$ be a bounded opesn set of $R^{n}$ with a smooth boundary $\Gamma$, which is a $C^{\infty}$-manifold of dimension $(n-1)$. Locally, $\Omega$ is totally on one side of $\Gamma$. We define the infinite order Sobolev space $W^{\infty}\left\{a_{\alpha}, 2\right\}(\Omega)$ of infinite order of periodic functions $\phi(x)$ defined on $\Omega$ [17-19] as follows:

$$
W^{\infty}\left\{a_{\alpha}, 2\right\}(\Omega)=\left\{(x) \in C^{\infty}(\Omega): \sum_{|\alpha|=0}^{\infty} a_{\alpha}\left\|D_{\alpha}\right\|_{2}^{2}<\infty\right\}
$$

where $C^{\infty}(\Omega)$ is the space of infinite differentiable functions, $a_{\alpha} \geq 0$ is a numerical sequence and $\|.\|_{2}$ is the canonical norm in the space $L^{2}(\Omega)$, and

$$
D^{\alpha}=\frac{\partial^{|\alpha|}}{\left(\partial x_{1}\right)^{\alpha_{1}} \cdots\left(\partial x_{n}\right)^{\alpha_{n}}}
$$

$\alpha=\left(\alpha_{1}, \cdots, \alpha_{n}\right)$ being a multi-index for differentiation, 
$|\alpha|=\sum_{i=1}^{n} \alpha_{i}$.

The space $W^{\infty}\left\{a_{\alpha}, 2\right\}(\Omega)$ is defined as the formal conjugate space to the space $W^{\infty}\left\{a_{\alpha}, 2\right\}(\Omega)$, namely:

$$
\begin{aligned}
& W^{\infty}\left\{a_{\alpha}, 2\right\}(\Omega) \\
& =\left\{\psi(x): \psi(x)=\sum_{|\alpha|=0}^{\infty}(-1)^{|\alpha|} a_{\alpha} D^{\alpha} \psi_{\alpha}(x)\right\}
\end{aligned}
$$

where $\psi_{\alpha} \in L^{2}(\Omega)$ and $\sum_{|\alpha|=0}^{\infty} a_{\alpha}\left\|\psi_{\alpha}\right\|_{2}^{2}<\infty$.

The duality pairing of the spaces $W^{\infty}\left\{a_{\alpha}, 2\right\}(\Omega)$ and $W^{-\infty}\left\{a_{\alpha}, 2\right\}(\Omega)$ is postulated by the formula

$$
(\phi, \psi)=\sum_{|\alpha|=0}^{\infty} a_{\alpha} \int_{\Omega} \psi_{\alpha}(x) D^{\alpha} \phi(x) \mathrm{d} x
$$

where

$$
\phi \in W^{\infty}\left\{a_{\alpha}, 2\right\}(\Omega), \psi \in W^{-\infty}\left\{a_{\alpha}, 2\right\}(\Omega)
$$

From above, $W^{\infty}\left\{a_{\alpha}, 2\right\}(\Omega)$ is everywhere dense in $L^{2}(\Omega)$ with topological inclusions and $W^{-\infty}\left\{a_{\alpha}, 2\right\}(\Omega)$ denotes the topological dual space with respect to $L^{2}(\Omega)$, so we have the following chain of inclusions:

$$
W^{\infty}\left\{a_{\alpha}, 2\right\}(\Omega) \subseteq L^{2}(\Omega) \subseteq W^{-\infty}\left\{a_{\alpha}, 2\right\}(\Omega)
$$

We now introduce $L^{2}\left(0, T ; L^{2}(\Omega)\right)$ which we shall denoted by $L^{2}(Q)$, where $\left.Q=\Omega \times\right] 0, T[$, denotes the space of measurable functions $t \rightarrow \phi(t)$ such that

$$
\|\phi\|_{L^{2}(Q)}=\left(\int_{0}^{T}\|\phi(t)\|_{2}^{2} \mathrm{~d} t\right)^{\frac{1}{2}}<\infty
$$

endowed with the scalar product $(f, g)=\int_{0}^{T}(f(t), g(t))_{L^{2}(\Omega)} \mathrm{d} t, L^{2}(Q)$ is a Hilbert space.

In the same manner we define the spaces $L^{2}\left(0, T ; W^{\infty}\left\{a_{\alpha}, 2\right\}(\Omega)\right)$, and $L^{2}\left(0, T ; W^{-\infty}\left\{a_{\alpha}, 2\right\}(\Omega)\right)$, as its formal conjugate.

Also, we have the following chain of inclusions:

$$
\begin{aligned}
& L^{2}\left(0, T ; W^{\infty}\left\{a_{\alpha}, 2\right\}(\Omega)\right) \subseteq L^{2}(Q) \\
& \subseteq L^{2}\left(0, T ; W^{-\infty}\left\{a_{\alpha}, 2\right\}(\Omega)\right)
\end{aligned}
$$

The construction of the Cartesian product of $n$-times to the above Hilbert spaces can be construct, for example

$$
\begin{aligned}
& \left(W^{\infty}\left\{a_{\alpha}, 2\right\}(\Omega)\right)^{n} \\
& =\underbrace{W^{\infty}\left\{a_{\alpha}, 2\right\}(\Omega) \times W^{\infty}\left\{a_{\alpha}, 2\right\}(\Omega) \times \cdots \times W^{\infty}\left\{a_{\alpha}, 2\right\}(\Omega)}_{n \text {-times }} \\
& =\prod_{i=1}^{n}\left(W^{\infty}\left\{a_{\alpha}, 2\right\}(\Omega)\right)^{i}
\end{aligned}
$$

with norm defined by:

$$
\|\phi\|_{\left(W^{\infty}\left\{a_{\alpha}, 2\right\}(\Omega)\right)^{n}}=\sum_{i=1}^{n}\left\|\phi_{i}\right\|_{W^{\infty}\left\{a_{\alpha}, 2\right\}(\Omega)}
$$

where $\phi=\left(\phi_{1}, \phi_{2}, \cdots, \phi_{n}\right)=\left(\phi_{i}\right)_{i=1}^{n}$ is a vector function and $\phi_{i} \in W^{\infty}\left\{a_{\alpha}, 2\right\}(\Omega)$.

Finally, we have the following chain of inclusions:

$$
\begin{aligned}
& \left(L^{2}\left(0, T ; W^{\infty}\left\{a_{\alpha}, 2\right\}(\Omega)\right)\right)^{n} \subseteq\left(L^{2}(Q)\right)^{n} \\
& \subseteq\left(L^{2}\left(0, T ; W^{-\infty}\left\{a_{\alpha}, 2\right\}(\Omega)\right)\right)^{n}
\end{aligned}
$$

where $\left(L^{2}\left(0, T ; W^{-\infty}\left\{a_{\alpha}, 2\right\}(\Omega)\right)\right)^{n}$ are the dual spaces of $\left(L^{2}\left(0, T ; W^{\infty}\left\{a_{\alpha}, 2\right\}(\Omega)\right)\right)^{n}$. The spaces considered in this paper are assumed to be real.

\section{Mixed Neumann Problem for Infinite Order Hyperbolic System Involving Time Lags}

The object of this section is to formulate the following mixed initial boundary value Neumann problem for infinite order hyperbolic system involving time lags which defines the state of the system model.

$$
\begin{aligned}
& \frac{\partial^{2} y}{\partial t^{2}}+A(t) y(x, t)+b(x, t) y(x, t-h)=u, \\
& (x, t) \in \Omega \times(0, T) \\
& y\left(x, t^{\prime}\right)=\Phi_{0}\left(x, t^{\prime}\right),\left(x, t^{\prime}\right) \in \Omega \times(-h, 0) \\
& y(x, 0)=y_{0}(x), x \in \Omega \\
& y^{\prime}(x, 0)=y_{1}(x), x \in \Omega \\
& \frac{\partial y}{\partial v_{A}}=c(x, t) y(x, t-h)+v,(x, t) \in \Gamma \times(0, T) \\
& y\left(x, t^{\prime}\right)=\Psi_{0}\left(x, t^{\prime}\right),\left(x, t^{\prime}\right) \in \Gamma \times(-h, 0)
\end{aligned}
$$

where $\Omega \subset R^{n}$ has the same properties as in Section 1 . We have

$$
\begin{aligned}
& y \equiv y(x, t ; v), y_{0} \equiv y(x, 0 ; v), y(T) \equiv y(x, T ; v), \\
& u \equiv u(x, t), v \equiv v(x, t) \\
& Q=\Omega \times(0, T), Q=\Omega \times[0, T], Q_{0}=\Omega \times(-h, 0), \\
& \Sigma=\Gamma \times(0, T), \Sigma_{0}=\Gamma \times(-h, 0),
\end{aligned}
$$

- $T$ is a specified positive number representing a finite time horizon;

- $h$ is a specific positive number representing a time lag;

- $b, c$ are given real $C^{\infty}$ functions defined on $\bar{Q}$, $\Sigma$ respectively;

- $y$ is a function defined on $Q$ such that 
$\Omega \times(0, T) \ni(x, t) \rightarrow y(x, t) \in R ;$

- $u, v$ are functions defined on $Q$ and $\Sigma$ such that $\Omega \times(0, T) \ni(x, t) \rightarrow u(x, t) \in R$ and $\Gamma \times(0, T) \ni(x, t) \rightarrow v(x, t) \in R$

- $\Phi_{0}, \Psi_{0}$ are initial functions defined on $Q_{0}, \Sigma_{0}$ such that

$$
\begin{aligned}
& \Omega \times(-h, 0) \ni\left(x, t^{\prime}\right) \rightarrow \Phi_{0}\left(x, t^{\prime}\right) \in R . \\
& \Gamma \times(-h, 0) \ni\left(x, t^{\prime}\right) \rightarrow \Psi_{0}\left(x, t^{\prime}\right) \in R .
\end{aligned}
$$

The hyperbolic operator $\frac{\partial^{2}}{\partial t^{2}}+A(t)$ in the state Equation (1) is an infinite order hyperbolic operator and $A(t)$ [19] is given by:

$$
A y=\sum_{|\alpha|=0}^{\infty}(-1)^{|\alpha|} a_{\alpha} D^{2|\alpha|} y(x, t),
$$

and

$$
A=\sum_{|\alpha|=0}^{\infty}(-1)^{|\alpha|} a_{\alpha} D^{2|\alpha|}
$$

is an infinite order self-adjoint elliptic partial differential operator maps $W^{\infty}\left\{a_{\alpha}, 2\right\}(\Omega)$ onto $W^{-\infty}\left\{a_{\alpha}, 2\right\}(\Omega)$.

For this operator we define the bilinear form as follows:

Definition 2.1. For each $t \in(0, T) R$, we define a family of bilinear forms on $W^{\infty}\left\{a_{\alpha}, 2\right\}(\Omega)$ by:

$$
\pi(t ; y, \phi)=(A(t) y, \phi)_{L^{2}(\Omega)}, \quad y, \phi \in W^{\infty}\left\{a_{\alpha}, 2\right\}(\Omega)
$$

where $A(t)$ maps $W^{\infty}\left\{a_{\alpha}, 2\right\}(\Omega)$ onto $W^{-\infty}\left\{a_{\alpha}, 2\right\}(\Omega)$ and takes the above form. Then

$$
\begin{aligned}
\pi(t ; y, \phi) & =(A(t) y, \phi)_{L^{2}(\Omega)} \\
& =\left(\sum_{|\alpha|=0}^{\infty}(-1)^{|\alpha|} a_{\alpha} D^{2|\alpha|} y(x, t), \phi(x)\right)_{L^{2}(\Omega)} \\
& =\int_{\Omega} \sum_{|\alpha|=0}^{\infty} a_{\alpha} D^{|\alpha|} y(x) D^{|\alpha|} \phi(x) \mathrm{d} x
\end{aligned}
$$

Lemma 2.1. The bilinear form $\pi(t ; y, \phi)$ is coercive on $W^{\infty}\left\{a_{\alpha}, 2\right\}(\Omega)$ that is

$$
\pi(t ; y, y) \geq \lambda\|y\|_{W^{\infty}\{a, 2\}(\Omega)}^{2}, \quad \lambda>0
$$

Proof. It is well known that the ellipticity of $A(t)$ is sufficient for the coerciveness of $\pi(t ; y, \phi)$ on $W^{\infty}\left\{a_{\alpha}, 2\right\}(\Omega)$.

$$
\pi(t ; \phi, \psi)=\int_{\Omega} \sum_{\|=0}^{\infty} a_{\alpha} D^{|\alpha|} \phi D^{|\alpha|} \psi \mathrm{d} x
$$

Then

$$
\begin{aligned}
& \pi(t ; y, y)=\int_{\Omega|\alpha|=0} \sum_{\alpha}^{\infty} a_{\alpha} D^{|\alpha|} y D^{|\alpha|} y \mathrm{~d} x \\
& \geq \sum_{\|=0}^{\infty} a_{\alpha}\left\|D^{2|\alpha|} y(x)\right\|_{L^{2}(\Omega)}^{2} \geq \lambda\|y\|_{W^{\infty}\left\{a_{\alpha}, 2\right\}(\Omega)}^{2}, \lambda>0
\end{aligned}
$$

Also we have:

$$
\begin{aligned}
& \forall y, \in W^{\infty}\left\{a_{\alpha}, 2\right\}(\Omega) \\
& \text { the function } t \rightarrow \pi(t ; y,) \\
& \text { is continuously differentiable in }(0, T) \\
& \text { and } \pi(t ; y, \phi)=\pi(t ; \phi, y)
\end{aligned}
$$

Equations (1)-(6) constitute a Neumann problem. Then the left-hand side of the boundary condition (5) may be written in the following form:

$$
\begin{aligned}
& \frac{\partial y(u)}{\partial v_{A}}=\sum_{|\omega|=0}^{\infty}\left(D^{\omega} y(u)\right) \cos \left(n, x_{k}\right)=q(x, t) \\
& x \in \Gamma, t \in(0, T)
\end{aligned}
$$

where $\frac{\partial}{\partial v_{A}}$ is a normal derivative at $\Gamma$, directed towards the exterior of $\Omega$, and $\cos \left(n, x_{k}\right)$ is the $k$-th direction cosine of $n$, with $n$ being the normal at $\Gamma$ exterior to $\Omega$.

Then (5) can be written as:

$$
\left.\begin{array}{l}
q(x, t)=c(x, t) y(x, t-h)+v(x, t), \\
x \in \Gamma, t \in(0, T) .
\end{array}\right\}
$$

We shall formulate sufficient conditions for the existence of a unique solution of the mixed boundary value problem (1)-(6) for the case where the boundary control $v \in L^{2}(\Sigma)$. For this purpose we introduce the Sobolev space $W^{\infty, 2}(Q)$ [20] (p. 6) defined by:

$$
\begin{aligned}
& W^{\infty, 2}(Q) \\
& =L^{2}\left(0, T ; W^{\infty}\left\{a_{\alpha}, 2\right\}(\Omega)\right) \cap W^{2}\left(0, T ; L^{2}(\Omega)\right)
\end{aligned}
$$

which is a Hilbert space normed by

$$
\begin{aligned}
& \|y\|_{W^{\infty, 2}(Q)}=\left[\int_{0}^{T}\|y\|_{W^{\infty}\left\{a_{\alpha}, 2\right\}(\Omega)}^{2} \mathrm{~d} t+\|y\|_{W^{2}\left(0, T ; L^{2}(\Omega)\right)}^{2}\right]^{1 / 2} \\
& =\left[\int_{Q}\left(\sum_{\|=0}^{\infty} a_{\alpha}\left|D^{\alpha} y\right|^{2}+\left|\frac{\partial y}{\partial t}\right|^{2}\right) \mathrm{d} x \mathrm{~d} t\right]^{1 / 2} \\
& =\left[\int_{Q}\left(a_{0}|y|^{2}+\sum_{|\alpha|=1}^{\infty} a_{\alpha}\left|D^{\alpha} y\right|^{2}+\left|\frac{\partial y}{\partial t}\right|^{2}\right) \mathrm{d} x \mathrm{~d} t\right]^{1 / 2}
\end{aligned}
$$

where the space $W^{2}\left(0, T ; L^{2}(\Omega)\right)$ denotes the Sobolev space of second order of functions defined on $(0, T)$ and taking values in $L^{2}(\Omega)$ [20] .

The existence of a unique solution for the mixed initial-boundary value problem (1)-(6) on the cylinder $Q$ can be proved using a constructive method, i.e., solving at first Equations (1)-(6) on the sub-cylinder $Q_{1}$ and in turn on $Q_{2}$ etc., until the procedure covers the whole cylinder $Q$. In this way, the solution in the previous step determines the next one. 
For simplicity, we introduce the following notation:

$$
\begin{gathered}
E_{j} \triangleq((j-1) h, j h), \quad Q_{j}=\Omega \times E_{j}, \quad \Sigma_{j}=\Gamma \times E_{j} \\
Q_{0}=\Omega \times(-h, 0), \quad \Sigma_{0}=\Gamma \times(-h, 0) \text { for } j=1, \cdots
\end{gathered}
$$

Using Theorem 6.1 of [20] (Vol. 2, p. 33), then the following result holds.

Theorem 2.1. Let $y_{0}, y_{1}, \Phi_{0}, \Psi_{0}, v$ and $u$ be given with $y_{0} \in W^{\infty}\left\{a_{\alpha}, 2\right\}(\Omega), y_{1} \in W^{\infty}\left\{a_{\alpha}, 2\right\}(\Omega)$, $\Phi_{0} \in W^{\infty, 2}\left(Q_{0}\right), \quad \Psi_{0} \in L^{2}\left(\Sigma_{0}\right), \quad v \in L^{2}(\Sigma)$ and $u \in W^{-\infty,-2}(Q)$ and the following compatibility relations:

$$
\begin{aligned}
& \frac{\partial y_{0}}{\partial v_{A}}(x, 0)=q_{1}(x, 0) \text { on } \Gamma \\
& \frac{\partial y_{1}}{\partial v_{A}}(x, 0)+\left(\frac{\partial}{\partial t}\left(\frac{\partial}{\partial v_{A}}\right)\right) y_{0}(x, 0) \\
& =\frac{\partial}{\partial t} q_{1}(x, 0) \text { on } \Gamma
\end{aligned}
$$

Then, there exists a unique solution $y \in W^{\infty, 2}(Q)$ for the mixed initial-boundary value problem (1)-(6). Moreover, $y\left(., t_{j}\right) \in W^{\infty}\left\{a_{\alpha}, 2\right\}(\Omega)$

$$
y^{\prime}\left(., t_{j}\right) \in W^{\infty}\left\{a_{\alpha}, 2\right\}(\Omega) \text {, for } j=1, \cdots, K .
$$

\section{Problem Formulation and Optimization Theorems}

Now, we formulate the optimal control problem for (1)(6) in the context of the Theorem 2.1, that is $v \in L^{2}(\Sigma)$.

Let us denote by $U=L^{2}(\Sigma)$ the space of controls. The time horizon $T$ is fixed in our problem.

The performance functional is given by:

$$
\begin{aligned}
I(v) & =\lambda_{1} \int_{Q}\left[y(x, t ; v)-z_{d}\right]^{2} \mathrm{~d} x \mathrm{~d} t \\
& +\lambda_{2} \int_{\Sigma}(N v) v d \Gamma \mathrm{d} t
\end{aligned}
$$

where $\lambda_{i} \geq 0$, and $\lambda_{1}+\lambda_{2}>0, \quad z_{d}$ is a given element in $t^{2}(Q) ; N$ is a positive linear operator on $L^{2}(\Sigma)$ into $L^{2}(\Sigma)$.

Control constraints: We define the set of admissible controls $U_{a d}$ such that

$$
U_{a d} \text { is closed, convex subset of } U=L^{2}(\Sigma)
$$

Let $y(x, t ; v)$ denote the solution of the mixed initialboundary value problem (1)-(6) at $(x, t)$ corresponding to a given control $v \in U_{a d}$. We note from Theorem 2.1 that for any $v \in U_{a d}$ the performance functional (15) is well-defined since $y(v) \in W^{\infty, 2}(Q) \subset L^{2}(Q)$.

Making use of the Loins's scheme we shall derive the necessary and sufficient conditions of optimality for the optimization problem (1)-(6), (15), (16). The solving of the formulated optimal control problem is equivalent to seeking a $v^{*} \in U_{a d}$ such that

$$
I\left(v^{*}\right) \leq I(v), \forall v \in U_{a d}
$$

From the Lion's scheme [21] (Theorem 1.3 of, p. 10), it follows that for $\lambda_{2}>0$ a unique optimal control $v^{*}$ exists. Moreover, $v^{*}$ is characterized by the following condition:

$$
I^{\prime}\left(v^{*}\right)\left(v-v^{*}\right) \geq 0 \quad \forall v \in U_{a d}
$$

For the performance functional of form (15) the relation (17) can be expressed as

$$
\begin{aligned}
& \lambda_{1} \int_{Q}\left(y\left(v^{*}\right)-z_{d}\right)\left[y(v)-y\left(v^{*}\right)\right] \mathrm{d} x \mathrm{~d} t \\
& +\lambda_{2} \int_{\Sigma} N v^{*}\left(v-v^{*}\right) \mathrm{d} \Gamma \mathrm{d} t \geq 0 \quad \forall v \in U_{a d}
\end{aligned}
$$

In order to simplify (18), we introduce the adjoint equation, and for every $v \in U_{a d}$, we define the adjoint variable $p=p(v) \equiv p(x, t ; v)$ as the solution of the equations:

$$
\begin{aligned}
& \frac{\partial^{2} p(v)}{\partial t^{2}}+A^{*}(t) p(v)+b(x, t+h) p(x, t+h ; v) \\
= & \lambda_{1}\left(y(v)-z_{d}\right),(x, t) \in \Omega \times(0, T-h) \\
& \frac{\partial^{2} p(v)}{\partial t^{2}}+A^{*}(t) p(v) \\
= & \lambda_{1}\left(y(v)-z_{d}\right),(x, t) \in \Omega \times(T-h, T) \\
& p(x, T ; v)=0, \quad x \in \Omega \\
& p^{\prime}(x, T ; v)=0, \quad x \in \Omega \\
& \frac{\partial p(v)}{\partial v_{A^{*}}}(x, t)=c(x, t+h) p(x, t+h ; v) \\
& (x, t) \in \Gamma \times(0, T-h) \\
& \frac{\partial p(v)}{\partial v_{A^{*}}}(x, t)=0, \quad(x, t) \in \Gamma \times(T-h, T)
\end{aligned}
$$

where $\frac{\partial p(v)}{\partial v_{A^{*}}}(x, t)=\sum_{|\omega|=0}^{\infty}\left(D^{\omega} p(v)\right) \cos \left(n, x_{\omega}\right)$

$$
A^{*}(t) p(v)=\sum_{|\alpha|=0}^{\infty}(-1)^{|\alpha|} a_{\alpha} D^{2|\alpha|} p(x, t)
$$

As in the above section with change of variables, i.e. with reversed sense of time. i.e., $t^{\prime}=T-t$, for given $z_{d} \in L^{2}(Q)$ and any $v \in L^{2}(\Sigma)$, there exists a unique solution $p(v) \in W^{\infty, 2}(Q)$ for problem (19)-(24).

The existence of a unique solution for the problem (19)-(24) on the cylinder $Q$ can be proved using a constructive method. It is easy to notice that for given $z_{d}$ and $v$, the problem (19)-(24) can be solved backwards in time starting from $t=T$, i.e. first solving (19)-(24) on the sub-cylinder $Q_{K}$ and in turn on $Q_{K-1}$, etc. until the 
procedure covers the whole cylinder $Q$. For this purpose, we may apply Theorem 2.1 (with an obvious change of variables). Hence, using Theorem 2.1, the following result can be proved.

Lemma 3.1. Let the hypothesis of Theorem 2.1 be satisfied. Then for given $z_{d} \in L^{2}\left(\Omega, R^{\infty}\right)$ and any $v \in L^{2}(\Sigma)$, there exists a unique solution

$p(v) \in W^{\infty, 1}(Q)$ for the adjoint problem (19)-(24).

We simplify (18) using the adjoint equation (19)-(24). For this purpose denoting by $p(0) \equiv p(x, 0 ; v)$ and $p(T) \equiv p(x, T ; v)$ respectively, setting $v=v^{*}$ in (19)(24), multiplying both sides of (19), (20) by $y(v)-y\left(v^{*}\right)$, then integrating over $\Omega \times(0, T-h)$ and $\Omega \times(T-h, T)$ respectively and then adding both sides of (19), (20), we get

$$
\begin{aligned}
& \lambda_{1} \int_{Q}\left(y\left(v^{*}\right)-z_{d}\right)\left[y(v)-y\left(v^{*}\right)\right] \mathrm{d} x \mathrm{~d} t \\
= & \int_{0}^{T} \int_{\Omega}\left(\frac{\partial^{2} p\left(v^{*}\right)}{\partial t^{2}}+A^{*}(t) p\left(v^{*}\right)\right] \times\left[y(v)-y\left(v^{*}\right)\right] \mathrm{d} x \mathrm{~d} t \\
& +\int_{0}^{T-h} \int_{\Omega} b(x, t+h) p\left(x, t+h ; v^{*}\right) \times\left[y(v)-y\left(v^{*}\right)\right] \mathrm{d} x \mathrm{~d} t \\
= & \int_{\Omega} p^{\prime}(x, t ; v) \times\left[y(v)-y\left(v^{*}\right)\right] \mathrm{d} x \\
& +\int_{0}^{T} \int_{\Omega} p\left(v^{*}\right) \frac{\partial^{2}}{\partial t^{2}}\left[y(v)-y\left(v^{*}\right)\right] \mathrm{d} x \mathrm{~d} t \\
& +\int_{0}^{T} \int_{\Omega} A^{*}(t) p\left(v^{*}\right)\left[y(x, t ; v)-y\left(x, t ; v^{*}\right)\right] \mathrm{d} x \mathrm{~d} t \\
& +\int_{0}^{T-h} \int_{\Omega} b(x, t+h) p\left(x, t+h ; v^{*}\right) \\
& \times\left[y(x, t ; v)-y\left(x, t ; v^{*}\right)\right] \mathrm{d} x \mathrm{~d} t
\end{aligned}
$$

Using the Equation (1), the second integral on the right-hand side of (26) can be written as

$$
\begin{aligned}
& \int_{0}^{T} \int_{\Omega} p\left(v^{*}\right)^{2} t^{2}\left[y(v)-y\left(v^{*}\right)\right] \mathrm{d} x \mathrm{~d} t \\
& =-\int_{0}^{T} \int_{\Omega} p\left(v^{*}\right) A(t)\left[y(v)-y\left(v^{*}\right)\right] \mathrm{d} x \mathrm{~d} t \\
& -\int_{0}^{T} \int_{\Omega} b(x, t) p\left(x, t ; v^{*}\right) \\
& \times\left[y(x, t-h ; v)-y\left(x, t-h ; v^{*}\right)\right] \mathrm{d} x \mathrm{~d} t \\
& =-\int_{0}^{T} \int_{\Omega} p\left(v^{*}\right) A(t)\left[y(v)-y\left(v^{*}\right)\right] \mathrm{d} x \mathrm{~d} t \\
& -\int_{-h}^{T-h} \int_{\Omega} b\left(x, t^{\prime}+h\right) p\left(x, t^{\prime}+h ; v^{*}\right) \\
& \times\left[y\left(x, t^{\prime} ; v\right)-y\left(x, t^{\prime} ; v^{*}\right)\right] \mathrm{d} x \mathrm{~d} t
\end{aligned}
$$

Using Green's formula, the third integral on the righthand side of (26) can be written as

$$
\begin{aligned}
& \int_{0}^{T} \int_{\Omega} A^{*}(t) p\left(v^{*}\right)\left[y(v)-y\left(v^{*}\right)\right] \mathrm{d} x \mathrm{~d} t \\
& =\int_{0}^{T} \int_{\Omega} p\left(v^{*}\right) A(t)\left[y(v)-y\left(v^{*}\right)\right] \mathrm{d} x \mathrm{~d} t \\
& +\int_{0}^{T} \int_{\Gamma} p\left(v^{*}\right)\left(\frac{\partial y(v)}{\partial v_{A}}-\frac{\partial y\left(v^{*}\right)}{\partial v_{A}}\right) \mathrm{d} \Gamma \mathrm{d} t \\
& -\int_{0}^{T} \int_{\Gamma} \frac{\partial p\left(v^{*}\right)}{\partial v_{A^{*}}}\left[y(v)-y\left(v^{*}\right)\right] \mathrm{d} \Gamma \mathrm{d} t
\end{aligned}
$$

Using the boundary condition (5), one can transform the second integral on the right-hand side of (28) into the form:

$$
\begin{aligned}
& \int_{0}^{T} \int_{\Gamma} p\left(v^{*}\right)\left(\frac{\partial y(v)}{\partial v_{A}}-\frac{\partial y\left(v^{*}\right)}{\partial v_{A}}\right) \mathrm{d} \Gamma \mathrm{d} t \\
& =\int_{0}^{T} \int_{\Gamma} p\left(x, t ; v^{*}\right) c(x, t) \times\left[y(x, t-h ; v)-y\left(x, t-h ; v^{*}\right)\right] \mathrm{d} \Gamma \mathrm{d} t \\
& \quad+\int_{0}^{T} \int_{\Gamma} p\left(x, t ; v^{*}\right)\left(v-v^{*}\right) \mathrm{d} \Gamma \mathrm{d} t \\
& =\int_{-h}^{T-h} \int_{\Gamma} p\left(x, t^{\prime}+h ; v^{*}\right) c\left(x, t^{\prime}+h\right) \\
& \quad \times\left[y\left(x, t^{\prime} ; v\right)-y\left(x, t^{\prime} ; v^{*}\right)\right] \mathrm{d} \Gamma \mathrm{d} t^{\prime} \\
& \quad+\int_{0}^{T} \int_{\Gamma} p\left(x, t ; v^{*}\right)\left(v-v^{*}\right) \mathrm{d} \Gamma \mathrm{d} t
\end{aligned}
$$

The last component in (28) can be rewritten as

$$
\begin{aligned}
& \int_{0}^{T} \int_{\Gamma} \frac{\partial p\left(v^{*}\right)}{\partial v_{A^{*}}}\left[y(v)-y\left(v^{*}\right)\right] \mathrm{d} \Gamma \mathrm{d} t \\
& =\int_{0}^{T-h} \int_{\Gamma} \frac{\partial p\left(v^{*}\right)}{\partial v_{A^{*}}}\left[y(v)-y\left(v^{*}\right)\right] \mathrm{d} \Gamma \mathrm{d} t \\
& +\int_{T-h}^{T} \int_{\Gamma} \frac{\partial p\left(v^{*}\right)}{\partial v_{A^{*}}}\left[y(v)-y\left(v^{*}\right)\right] \mathrm{d} \Gamma \mathrm{d} t
\end{aligned}
$$

Substituting (29) and (30) into (28), and then (27), (28) into (26), we obtain

$$
\begin{aligned}
& \lambda_{1} \int_{Q}\left(y\left(v^{*}\right)-z_{d}\right)\left[y(v)-y\left(v^{*}\right)\right] \mathrm{d} x \mathrm{~d} t \\
& =-\int_{0}^{T} \int_{\Omega} p\left(v^{*}\right) A(t)\left[y(v)-y\left(v^{*}\right)\right] \mathrm{d} x \mathrm{~d} t \\
& -\int_{-h}^{0} \int_{\Omega} p\left(x, t+h ; v^{*}\right) b(x, t+h) \times\left[y(x, t ; v)-y\left(x, t ; v^{*}\right)\right] \mathrm{d} x \mathrm{~d} t \\
& -\int_{0}^{T-h} \int_{\Omega} p\left(x, t+h ; v^{*}\right) b(x, t+h) \\
& \times\left[y(x, t ; v)-y\left(x, t ; v^{*}\right)\right] \mathrm{d} x \mathrm{~d} t \\
& +\int_{0}^{T} \int_{\Omega} p\left(v^{*}\right) A(t)\left[y(v)-y\left(v^{*}\right)\right] \mathrm{d} x \mathrm{~d} t \\
& +\int_{-h}^{0} \int_{\Gamma} p\left(x, t+h ; v^{*}\right) c(x, t+h) \times\left[y(x, t ; v)-y\left(x, t ; v^{*}\right)\right] \mathrm{d} \Gamma \mathrm{d} t \\
& +\int_{0}^{T-h} \int_{\Gamma} p\left(x, t+h ; v^{*}\right) c(x, t+h) \\
& \times\left[y(x, t ; v)-y\left(x, t ; v^{*}\right)\right] \mathrm{d} \Gamma \mathrm{d} t \\
& +\int_{0}^{T} \int_{\Gamma} p\left(x, t ; v^{*}\right)\left(v-v^{*}\right) \mathrm{d} \Gamma \mathrm{d} t \\
& -\int_{0}^{T-h} \int_{\Gamma} \frac{\partial p\left(v^{*}\right)}{\partial v}\left[y(v)-y\left(v^{*}\right)\right] \mathrm{d} \Gamma \mathrm{d} t \\
& -\int_{T-h}^{T} \int_{\Gamma} \frac{\partial p\left(v^{*}\right)}{\partial v A^{*}}\left[y(v)-y\left(v^{*}\right)\right] \mathrm{d} \Gamma \mathrm{d} t \\
& =\int_{0}^{T} \int_{\Gamma} p\left(x, t ; v^{*}\right)\left(v-v^{*}\right) \mathrm{d} \Gamma \mathrm{d} t
\end{aligned}
$$


Substituting (31) into (18) gives

$$
\int_{0}^{T} \int_{\Gamma}\left(p\left(v^{*}\right)+{ }_{2} N v^{*}\right)\left(v-v^{*}\right) \mathrm{d} \Gamma \mathrm{d} t \geq 0 \quad \forall v \in U_{a d}
$$

The foregoing result is now summarized.

Theorem 3.1. For the problem (1)-(6), with the performance functional (15) with $z_{d} \in L^{2}(Q)$ and $\lambda_{2}>0$ and with conditions (16), there exists a unique optimal control $v^{*}$ which satisfies the maximum condition (32).

\section{Mathematical Examples}

Example 3.1. Consider now the particular case where $U_{a d}=U=L^{2}(\Sigma)$ (no constraints case). Thus the maximum condition (32) is satisfied when

$$
v=-\lambda_{2} N^{-1} p\left(v^{*}\right)
$$

If $N$ is the identity operator on $L^{2}(\Sigma)$, then from the Lemma 3.1 follows that $v^{*} \in W^{\infty, 2}(Q)$.

Example 3.2. We can also consider an analogous optimal control problem where the performance functional is given by:

$$
\begin{aligned}
I(v) & =\lambda_{1} \int_{\Sigma}\left[\left.y(x, t ; v)\right|_{\Sigma}-z_{d}\right]^{2} \mathrm{~d} \Gamma \mathrm{d} t \\
& +\lambda_{2} \int_{\Sigma}(N v) v \mathrm{~d} \Gamma \mathrm{d} t
\end{aligned}
$$

where $z_{\Sigma d} \in L^{2}(\Sigma)$.

From Theorem 2.1 and the Trace Theorem [20] (Vol. 2, p. 9), for each $v \in L^{2}(\Sigma)$, there exists a unique solution $y(v) \in W^{\infty, 1}(Q)$ with $\left.y\right|_{\Sigma} \in L^{2}(\Sigma)$. Thus, $I(v)$ is well defined. Then, the optimal control $v^{*}$ is characterized by:

$$
\begin{aligned}
& \lambda_{1} \int_{\Sigma}\left(\left.y\left(v^{*}\right)\right|_{\Sigma}-z_{d}\right)\left[\left.y(v)\right|_{\Sigma}-\left.y\left(v^{*}\right)\right|_{\Sigma}\right] \mathrm{d} \Gamma \mathrm{d} t \\
& +\lambda_{2} \int_{\Sigma} N v^{*}\left(v-v^{*}\right) \mathrm{d} \Gamma \mathrm{d} t \geq 0 \quad \forall v \in U_{a d}
\end{aligned}
$$

We define the adjoint variable $p=p(v)=p(x, t ; v)$ as the solution of the equations:

$$
\begin{aligned}
& \frac{\partial^{2} p(v)}{\partial t^{2}}+A^{*}(t) p(v)+b(x, t+h) p(x, t+h ; v)=0, \\
& (x, t) \in \Omega \times(0, T-h), \\
& \frac{\partial^{2} p(v)}{\partial t^{2}}+A^{*}(t) p(v)=0,(x, t) \in \Omega \times(T-h, T) \\
& p(x, T ; v)=0, \quad x \in \Omega \\
& p^{\prime}(x, T ; v)=0, \quad x \in \Omega \\
& \frac{\partial p(v)}{\partial v_{A^{*}}}(x, t)=c(x, t+h) p(x, t+h ; v) \\
& +\lambda_{1}\left(\left.y(v)\right|_{\Sigma}(x, t)-z_{\Sigma d}\right),(x, t) \in \Gamma \times(0, T-h)
\end{aligned}
$$

$$
\begin{aligned}
& \frac{\partial p(v)}{\partial v_{A^{*}}}(x, t)=\lambda_{1}\left(\left.y(v)\right|_{\Sigma}(x, t)-z_{\Sigma d}\right) \\
& (x, t) \in \Gamma \times(T-h, T),
\end{aligned}
$$

As in the above section, we have the following result.

Lemma 3.2. Let the hypothesis of Theorem 2.1 be satisfied. Then, for given $z_{\Sigma d} \in L^{2}(\Sigma)$ and any $v \in L^{2}(\Sigma)$, there exists a unique solution $p\left(v^{*}\right) \in W^{\infty, 2}(Q)$ to the adjoint problem (35)-(40).

Using the adjoint Equations (35)-(40) in this case, the condition (34) can also be written in the following form

$$
\int_{0}^{T} \int_{\Gamma}\left(p\left(v^{*}\right)+\lambda_{2} N v^{*}\right)\left(v-v^{*}\right) \mathrm{d} \Gamma \mathrm{d} t \geq 0, \forall v \in U_{a d}
$$

The following result is now summarized.

Theorem 3.2. For the problem (1)-(6) with the performance function (33) with $z_{\Sigma d} \in L^{2}(\Sigma)$ and $\lambda_{2}>0$, and with constraint (16), and with adjoint Equations (35)-(40), there exists a unique optimal control $v^{*}$ which satisfies the maximum condition (41).

Example 3.3. Case: $u \in L^{2}(Q)$. We can also consider an analogous optimal control problem where the performance functional is given by:

$$
\begin{aligned}
I(u) & =\lambda_{1} \int_{Q}\left[y(x, t ; u)-z_{d}\right]^{2} \mathrm{~d} x \mathrm{~d} t \\
& +\lambda_{2} \int_{Q}(N u) u \mathrm{~d} x \mathrm{~d} t
\end{aligned}
$$

where $z_{d} \in L^{2}(Q)$.

From Theorem 2.1 and the Trace Theorem [20] (Vol. 2, p. 9), for each $u \in L^{2}(Q)$, there exists a unique solution $y(u) \in W^{\infty, 1}(Q)$. Thus, $I$ is well defined. Then, the optimal control $u^{*}$ is characterized by

$$
\begin{aligned}
& \lambda_{1} \int_{Q}\left(y\left(u^{*}\right)-z_{d}\right)\left[y(u)-y\left(u^{*}\right)\right] \mathrm{d} x \mathrm{~d} t \\
& +\lambda_{2} \int_{Q} N u^{*}\left(u-u^{*}\right) \mathrm{d} x \mathrm{~d} t \geq 0 \quad \forall u \in U_{a d}
\end{aligned}
$$

We define the adjoint variable $p=p(u)=p(x, t ; u)$ as the solution of the equations:

$$
\begin{aligned}
& \frac{\partial^{2} p(u)}{\partial t^{2}}+A^{*}(t) p(u)+b(x, t+h) p(x, t+h ; u)=0 \\
& (x, t) \in \Omega \times(0, T-h) \\
& \frac{\partial^{2} p(u)}{\partial t^{2}}+A^{*}(t) p(u)=0,(x, t) \in \Omega \times(T-h, T) \\
& p(x, T ; u)=0, \quad x \in \Omega \\
& p^{\prime}(x, T ; u)=\lambda_{1}\left(y(u)(x, t)-z_{d}\right), \quad x \in \Omega \\
& \frac{\partial p(u)}{\partial v_{A}^{*}}(x, t)=c(x, t+h) p(x, t+h ; u) \\
& (x, t) \in \Gamma \times(0, T-h)
\end{aligned}
$$




$$
p(u) v_{A^{*}}(x, t)=0,(x, t) \in \Gamma \times(T-h, T)
$$

As in the above section, we have the following result.

Lemma 3.3. Let the hypothesis of Theorem 2.1 be satisfied. Then, for given $z_{d} \in L^{2}(Q)$ and any

$u \in L^{2}(Q)$, there exists a unique solution

$p\left(u^{*}\right) \in W^{\infty, 2}(Q)$ to the adjoint problem (44)-(49).

Using the adjoint equations (44)-(49) in this case, the condition (43) can also be written in the following form:

$$
\int_{0}^{T} \int_{\Omega}\left(p\left(u^{*}\right)+{ }_{2} N u^{*}\right)\left(u-u^{*}\right) \mathrm{d} x \mathrm{~d} t \geq 0, \forall u \in U_{a d}
$$

The following result is now summarized.

Theorem 3.3. For the problem (1)-(6), (44)-(49), (16) with $z_{d} \in L^{2}(Q), \lambda_{2}>0$, there exists a unique optimal control $u^{*}$ which satisfies the maximum condition (50).

\section{Generalization}

The optimal control problems presented her can be extended to certain different two cases. Case 1: Optimal control for $2 \times 2$ coupled infinite order hyperbolic systems involving constant time lags. Case 2: Optimal control for $n \times n$ coupled infinite order hyperbolic systems involving constant time lags. Such extension can be applied to solving many control problems in mechanical engineering.

Case 1: Optimal control for $2 \times 2$ coupled infinite order hyperbolic systems involving constant time lags.

We will extend the discussions to study the optimal control for $2 \times 2$ coupled infinite order hyperbolic systems involving constant time lags. We consider the case where $v=\left(v_{1}, v_{2}\right) \in L^{2}(\Sigma) \times L^{2}(\Sigma)$, the performance functional is given by:

$$
\begin{aligned}
I(v) & =\sum_{i=1}^{2}\left(\lambda_{1} \int_{Q}\left[y_{i}(x, t ; v)-z_{i d}\right]^{2} \mathrm{~d} x \mathrm{~d} t\right. \\
& \left.+\lambda_{2} \int_{\Sigma}\left(N_{i} v_{i}\right) v_{i} \mathrm{~d} x \mathrm{~d} t\right)
\end{aligned}
$$

where $z_{d}=\left(z_{1 d}, z_{2 d}\right) \in\left(L^{2}(Q)\right)^{2}$.

The following results can now be proved.

Theorem 4.1. Let $y_{0}, y_{1}, \Phi_{0}, \Psi_{0}, v$ and $u$ be given with

$$
\begin{aligned}
& y_{0}=\left(y_{0,1}, y_{0,2}\right) \in\left(W^{\infty}\left\{a_{\alpha}, 2\right\}(\Omega)\right)^{2}, \\
& y_{1}=\left(y_{1,1}, y_{1,2}\right) \in\left(W^{\infty}\left\{a_{\alpha}, 2\right\}(\Omega)\right)^{2}, \\
& \Phi_{0}=\left(\Phi_{0,1}, \Phi_{0,2}\right) \in\left(W^{\infty, 2}\left(Q_{0}\right)\right)^{2}, \\
& \Psi_{0}=\left(\Psi_{0,1}, \Psi_{0,2}\right) \in\left(L^{2}\left(\Sigma_{0}\right)\right)^{2}, \\
& v=\left(v_{1}, v_{2}\right) \in\left(L^{2}\left(\sum\right)\right)^{2} \\
& \text { and } u=\left(u_{1}, u_{2}\right) \in\left(W^{-\infty,-2}(Q)\right)^{2} .
\end{aligned}
$$

Then, there exists a unique solution $y=\left(y_{1}, y_{2}\right) \in\left(W^{-\infty,-2}(Q)\right)^{2}$ for the following mixed initial-boundary value problem:

$$
\begin{aligned}
& \frac{\partial^{2} y_{1}}{\partial t^{2}}+\left(\sum_{|\alpha|=0}^{\infty}(-1)^{|\alpha|} a_{\alpha} D^{2 \alpha}+1\right) y_{1} \\
& +b_{1}(x, t) y_{1}(x, t-h)-y_{2}=u_{1}, \quad \text { in } Q \\
& \frac{\partial^{2} y_{2}}{\partial t^{2}}+\left(\sum_{|\alpha|=0}^{\infty}(-1)^{|\alpha|} a_{\alpha} D^{2 \alpha}+1\right) y_{2} \\
& \left.+b_{2}(x, t) y_{2}(x, t-h)+y_{1}=u_{2} \text {, in } Q\right) \\
& y_{1}\left(x, t^{\prime} ; u\right)=\Phi_{0,1}\left(x, t^{\prime}\right) \text {, } \\
& y_{2}\left(x, t^{\prime} ; u\right)=\Phi_{0,2}\left(x, t^{\prime}\right) \\
& \left(x, t^{\prime}\right) \in \Omega \times(-h, 0) \\
& y_{1}(x, 0 ; v)=y_{0,1}, y_{2}(x, 0 ; v)=y_{0,2}, x \in \Omega \\
& y_{1}^{\prime}(x, 0 ; v)=y_{1,1}, y_{2}^{\prime}(x, 0 ; v)=y_{1,2}, x \in \Omega \\
& \frac{\partial y_{1}}{\partial v_{A}}=c_{1}(x, t) y_{1}(x, t-h)+v_{1}, \quad \text { on } \Sigma, \\
& \left.\frac{\partial y_{2}}{\partial v_{A}}=c_{2}(x, t) y_{2}(x, t-h)+v_{2}, \quad \text { on } \Sigma\right\} \\
& y_{1}\left(x, t^{\prime} ; u\right)=\Psi_{0,1}\left(x, t^{\prime}\right), \quad\left(x, t^{\prime}\right) \in \Gamma \times(-h, 0) \\
& \left.y_{2}\left(x, t^{\prime} ; u\right)=\Psi_{0,2}\left(x, t^{\prime}\right), \quad\left(x, t^{\prime}\right) \in \Gamma \times(-h, 0)\right\}
\end{aligned}
$$

where

$$
\begin{aligned}
& y \equiv y(x, t ; v)=\left(y_{1}(x, t ; v), y_{2}(x, t ; v)\right) \in\left(W^{\infty, 2}(Q)\right)^{2} \\
& u \equiv u(x, t)=\left(u_{1}(x, t), u_{2}(x, t)\right) \in\left(W^{-\infty,-2}(Q)\right)^{2} \\
& v \equiv v(x, t)=\left(v_{1}(x, t), v_{2}(x, t)\right) \in\left(L^{2}(\Sigma)\right)^{2}
\end{aligned}
$$

Lemma 4.1. Let the hypothesis of Theorem 4.1 be satisfied. Then for given $z_{d}=\left(z_{1 d}, z_{2 d}\right) \in\left(L^{2}(Q)\right)^{2}$ and any $v=\left(v_{1}, v_{2}\right) \in\left(L^{2}(\Sigma)\right)^{2}$, there exists a unique solution $p(v)=\left(p_{1}(v), p_{2}(v)\right) \in\left(W^{\infty, 2}(Q)\right)^{2}$ for the adjoint problem:

$$
\left.\begin{array}{l}
\frac{\partial^{2} p_{1}(v)}{\partial t^{2}}+\left(\sum_{|\alpha|=0}^{\infty}(-1)^{|\alpha|} a_{\alpha} D^{2}+1\right) p_{1}(v) \\
+b_{1}(x, t+h) p_{1}(x, t+h ; v)+p_{2}(v)=\lambda_{1}\left(y_{1}(v)-z_{1 d}\right) \\
(x, t) \in \Omega \times(0, T-h), \\
\frac{\partial^{2} p_{1}(v)}{\partial t^{2}}+\left(\sum_{|\alpha|=0}^{\infty}(-1)^{|\alpha|} a_{\alpha} D^{2}+1\right) p_{2}(v)+b_{2}(x, t+h) \\
p_{2}(x, t+h ; v)-p_{1}(v)=\lambda_{1}\left(y_{2}(v)-z_{2 d}\right), \\
(x, t) \in \Omega \times(0, T-h)
\end{array}\right\}
$$




$$
\begin{aligned}
& \frac{\partial^{2} p_{1}(v)}{\partial t^{2}}+\left(\sum_{|\alpha|=0}^{\infty}(-1)^{|\alpha|} a_{\alpha} D^{2}+1\right) p_{1}(v)=0 \\
& x \in \Omega, t \in(T-h, T), \\
& \frac{\partial^{2} p_{2}(v)}{\partial t^{2}}+\left(\sum_{|\alpha|=0}^{\infty}(-1)^{|\alpha|} a_{\alpha} D^{2}+1\right) p_{2}(v)=0 \\
& (x, t) \in \Omega \times(T-h, T), \\
& p_{1}(x, T ; v)=0, \quad x \in \Omega, \\
& \left.p_{2}(x, T ; v)=0, \quad x \in \Omega,\right\} \\
& p_{1}^{\prime}(x, T ; v)=0, \quad x \in \Omega, \\
& \left.p_{2}^{\prime}(x, T ; v)=0, \quad x \in \Omega,\right\} \\
& \frac{\partial p_{1}(x, t)}{\partial v_{A^{*}}}=c_{1}(x, t+h) p_{1}(x, t+h ; v), \\
& (x, t) \in \Gamma \times(0, T-h) \\
& \frac{\partial p_{2}(x, t)}{\partial v_{A^{*}}}=c_{2}(x, t+h) p_{2}(x, t+h ; v), \\
& (x, t) \in \Gamma \times(0, T-h) \\
& \frac{\partial p_{1}(x, t)}{\partial v_{A^{*}}}=0, \quad(x, t) \in \Gamma \times(T-h, T), \\
& \frac{\partial p_{2}(x, t)}{\partial v_{A^{*}}}=0, \quad(x, t) \in \Gamma \times(T-h, T) .
\end{aligned}
$$

\section{Theorem 4.2. The optimal control}

$v^{*} \equiv v^{*}(x, t)=\left(v_{1}^{*}(x, t), v_{2}^{*}(x, t)\right) \in\left(L^{2}(\Sigma)\right)^{2}$ is characterized by the following maximum condition

$$
\begin{aligned}
& \int_{0}^{T} \int_{\Gamma}\left(\left[p_{1}\left(v^{*}\right)+{ }_{2} N_{1} v_{1}^{*}\right]\left(v_{1}-v_{1}^{*}\right)\right. \\
& \left.+\left[p_{2}\left(v^{*}\right)+{ }_{2} N_{2} v_{2}^{*}\right]\left(v_{2}-v_{2}^{*}\right)\right) \mathrm{d} \Gamma \mathrm{d} t \geq 0 \\
& \forall v=\left(v_{1}, v_{2}\right) \in\left(L^{2}(\Sigma)\right)^{2}
\end{aligned}
$$

where $p_{2}(x, t ; v) \in\left(W^{\infty, 2}(Q)\right)^{2}$ is the adjoint state.

The foregoing result is now summarized.

Theorem 4.3. For the problem (52)-(57) with the performance function (51) with

$z_{d}=\left(z_{1 d}, z_{2 d}\right) \in\left(L^{2}(Q)\right)^{2}$ and $\lambda_{2}>0$, and with constraint: $U_{a d}$ is closed, convex subset of $\left(L^{2}(\Sigma)\right)^{2}$, and with adjoint equations (58)-(63), then there exists a unique optimal control

$$
v^{*} \equiv v^{*}(x, t)=\left(v_{1}^{*}(x, t), v_{2}^{*}(x, t)\right) \in\left(L^{2}(\Sigma)\right)^{2}
$$

which satisfies the maximum condition (64).
Case 2: Optimal control for $n \times n$ coupled infinite order hyperbolic systems involving constant time lags.

We will extend the discussion to $n \times n$ coupled infinite order hyperbolic systems involving constant time lags. We consider the case where $v=\left(v_{1}, v_{2}, \cdots, v_{n}\right) \in\left(L^{2}(\Sigma)\right)^{n}$, the performance functional is given by (El-Saify, 2005; 2006):

$$
\begin{aligned}
I(v) & =\sum_{i=1}^{n}\left(\lambda_{1} \int_{Q}\left[y_{i}(x, t ; v)-z_{i d}\right]^{2} \mathrm{~d} x \mathrm{~d} t\right. \\
& \left.+\lambda_{2} \int_{\Sigma}\left(N_{i} v_{i}\right) v_{i} \mathrm{~d} x \mathrm{~d} t\right)
\end{aligned}
$$

where $z_{d}=\left(z_{1 d}, z_{2 d}, \cdots, z_{n d}\right) \in\left(L^{2}(Q)\right)^{n}$.

The following results can now be proved.

Theorem 4.4. Let $y_{0}, y_{1}, \Phi_{0}, \Psi_{0}, v$ and $u$ be given with

$$
\begin{aligned}
& y_{0}=\left(y_{0,1}, y_{0,2}, \cdots, y_{0, n}\right) \in\left(W^{\infty}\left\{a_{\alpha}, 2\right\}(\Omega)\right)^{n}, \\
& y_{1}=\left(y_{1,1}, y_{1,2}, \cdots, y_{1, n}\right) \in\left(W^{\infty}\left\{a_{\alpha}, 2\right\}(\Omega)\right)^{n}, \\
& \Phi_{0}=\left(\Phi_{0,1}, \Phi_{0,2}, \cdots, \Phi_{0, n}\right) \in\left(W^{\infty, 1}\left(Q_{0}\right)\right)^{n}, \\
& \Psi_{0}=\left(\Psi_{0,1}, \Psi_{0,2}, \cdots, \Psi_{0, n}\right) \in\left(L^{2}\left(\Sigma_{0}\right)\right)^{n}, \\
& v=\left(v_{1}, v_{2}, \cdots, v_{n}\right) \in\left(L^{2}(\Sigma)\right)^{n}
\end{aligned}
$$$$
\text { and } u=\left(u_{1}, u_{2}, \cdots, u_{n}\right) \in\left(W^{-\infty,-2}(Q)\right)^{n} \text {. }
$$

Then, there exists a unique solution $y=\left(y_{1}, y_{2}, \cdots, y_{n}\right) \in\left(W^{\infty, 2}(Q)\right)^{n}$ for the following mixed initial-boundary value problem: $\forall i, i=1,2, \cdots, n$ we have

$$
\begin{aligned}
& \frac{\partial^{2} y_{i}}{\partial t^{2}}+S(t) y_{i}(x, t)+b_{i}(x, t) y_{i}(x, t-h)=u_{i} \\
& (x, t) \in \Omega \times(0, T) \\
& y_{i}\left(x, t^{\prime}\right)=\Phi_{0, i}\left(x, t^{\prime}\right) \quad\left(x, t^{\prime}\right) \in \Omega \times(-h, 0) \\
& y_{i}(x, 0)=y_{0, i}(x), \quad x \in \Omega \\
& y_{i}^{\prime}(x, 0)=y_{1, i}(x), \quad x \in \Omega \\
& \frac{\partial y_{i}}{\partial v_{S}}=c_{i}(x, t) y_{i}(x, t-h)+v_{i} \\
& (x, t) \in \Gamma \times(0, T) \\
& y_{i}\left(x, t^{\prime}\right)=\Psi_{0, i}\left(x, t^{\prime}\right) \quad\left(x, t^{\prime}\right) \in \Gamma \times(-h, 0)
\end{aligned}
$$

where

$$
\begin{aligned}
& y \equiv y(x, t ; v)=\left(y_{1}(x, t ; v), y_{2}(x, t ; v), \cdots, y_{n}(x, t ; v)\right) \\
& \in\left(W^{\infty, 2}(Q)\right)^{n}
\end{aligned}
$$




$$
\begin{gathered}
u \equiv u(x, t)=\left(u_{1}(x, t), u_{2}(x, t), \cdots, u_{n}(x, t)\right) \\
\in\left(W^{-\infty,-2}(Q)\right)^{n} \\
v \equiv v(x, t)=\left(v_{1}(x, t), v_{2}(x, t), \cdots, v_{n}(x, t)\right) \in\left(L^{2}(\Sigma)\right)^{n}
\end{gathered}
$$$$
\boldsymbol{S}(t)=\left(\begin{array}{cccc}
\sum_{|\alpha|=0}^{\infty}(-1)^{|\alpha|} a_{\alpha} D^{2 \alpha}+1 & -1 & 1 & -1 \\
1 & \sum_{|\alpha|=0}^{\infty}(-1)^{|\alpha|} a_{\alpha} D^{2 \alpha}+1 & \cdots & -1 \\
\cdots & \cdots & \cdots & \cdots \\
\cdots & 1 & \cdots & \cdots \\
1 & & & \sum_{|\alpha|=0}^{\infty}(-1)^{|\alpha|} a_{\alpha} D^{2 \alpha}+1
\end{array}\right)_{n \times n}
$$

That is

$$
\sum_{|\alpha|=0}^{\infty}(-1)^{\alpha \|} a_{\alpha} D^{2} y_{i}(x)+\sum_{j=1}^{n} B_{i j} y_{j}(x), \forall i=1,2, \cdots, n
$$

where

$$
B_{i j}= \begin{cases}1 & \text { if } i \geq j \\ -1 & \text { if } \quad i<j\end{cases}
$$

Lemma 4.2. Let the hypothesis of Theorem 4.4 be satisfied. Then for given

$$
\begin{aligned}
& z_{d}=\left(z_{1 d}, z_{2 d}, \cdots, z_{n d}\right) \in\left(L^{2}(Q)\right)^{n} \text { and any } \\
& v(x, t)=\left(v_{1}(x, t), v_{2}(x, t), \cdots, v_{n}(x, t)\right) \in\left(L^{2}(\Sigma)\right)^{n} \text {, there } \\
& \text { exists a unique solution } \\
& p(v) \equiv p(x, t ; v)=\left(p_{1}(x, t ; v), p_{1}(x, t ; v), \cdots, p_{n}(x, t ; v)\right) \\
& \in\left(W^{\infty, 2}(Q)\right)^{n}
\end{aligned}
$$

for the adjoint problem: $\forall i, i=1,2, \cdots, n$, we have

$$
\left.\begin{array}{l}
\frac{\partial^{2} p_{i}(v)}{\partial t^{2}}+S^{*}(t) p_{i}(v)+b_{i}(x, t+h) p_{i}(x, t+h ; v) \\
=\lambda_{1}\left(y_{i}(v)-z_{i d}\right),(x, t) \in \Omega \times(0, T-h) \\
\frac{\partial^{2} p_{i}(v)}{\partial t^{2}}+S^{*}(t) p_{i}(v)=\lambda_{1}\left(y_{i}(v)-z_{i d}\right), \\
(x, t) \in \Omega \times(T-h, T) \\
p_{i}(x, T, v)=0, \quad x \in \Omega \\
p_{i}^{\prime}(x, T, v)=0, \quad x \in \Omega \\
\frac{\partial p_{i}(v)}{\partial v_{S^{*}}}(x, t)=c_{i}(x, t+h) p_{i}(x, t+h ; v), \\
(x, t) \in \Gamma \times(0, T-h),
\end{array}\right\}
$$

$$
\frac{\partial p_{i}(v)}{\partial v_{S^{*}}}(x, t)=0, \quad(x, t) \in \Gamma \times(T-h, T)
$$

Theorem 4.5. The optimal control

where

is the adjoint state.

The foregoing result is now summarized. performance function (65) with unique optimal control

which satisfies the maximum condition (79). $b_{i}, c_{i}$ are given real $C^{\infty}$ functions defined on $Q, \Sigma$, respectively, $h$ is a time lags,

$\Phi_{0, i}, \Psi_{0, i}$ are initial functions defined on $Q_{0}, \Sigma_{0}$ respectively.

The operator $\boldsymbol{S}(t)$ is an $n \times n$ matrix takes the form [22-25] (El-Saify \& Bahaa 2000; 2001; 2002; 2003).

$$
\begin{aligned}
v^{*} & \equiv v^{*}(x, t) \\
& =\left(v_{1}^{*}(x, t), v_{2}^{*}(x, t), \cdots, v_{n}^{*}(x, t)\right) \in\left(L^{2}(\Sigma)\right)^{n}
\end{aligned}
$$

is characterized by the following maximum condition

$$
\left.\begin{array}{l}
\sum_{i=1}^{n} \int_{0}^{T} \int_{\Gamma}\left(\left[p_{i}\left(v^{*}\right)+{ }_{2} N_{i} v_{i}^{*}\right]\left(v_{i}-v_{i}^{*}\right)\right) \mathrm{d} \Gamma \mathrm{d} t \geq 0, \\
\forall v=\left(v_{1}, v_{2}, \cdots, v_{n}\right) \in\left(U_{a d}\right)^{n},
\end{array}\right\}
$$

$$
\begin{aligned}
& p\left(v^{*}\right) \equiv p\left(x, t ; v^{*}\right) \\
& =\left(p_{1}\left(x, t ; v^{*}\right), p_{1}\left(x, t ; v^{*}\right), \cdots, p_{n}\left(x, t ; v^{*}\right)\right) \in\left(W^{\infty, 2}(Q)\right)^{n}
\end{aligned}
$$

Theorem 4.6. For the problem (66)-(71) with the

$z_{d}=\left(z_{1 d}, z_{2 d}, \cdots, z_{n d}\right) \in\left(L^{2}(Q)\right)^{n}$ and $\lambda_{2}>0$, and with constraint: $U_{a d}$ is closed, convex subset of $\left(L^{2}(\Sigma)\right)^{n}$, and with adjoint Equations (73)-(78), then there exists a

$$
v^{*} \equiv v^{*}(x, t)=\left(v_{1}^{*}(x, t), v_{2}^{*}(x, t), \cdots, v_{n}^{*}(x, t)\right) \in\left(L^{2}(\Sigma)\right)^{n}
$$

In the case of performance functionals $(15,33,42,51$ and 65) with $\lambda_{1}>0$ and $\lambda_{2}=0$, the optimal control problem reduces to minimization of the functional on a closed and convex subset in a Hilbert space. Then, the optimization problem is equivalent to a quadratic pro- 
gramming one, which can be solved by the use of the well-known Gilbert algorithm.

\section{Conclusions}

The optimization problem presented in the paper constitutes a generalization of the optimal boundary control problem of a second order hyperbolic systems involving constant time lags appearing in the boundary condition have been considered in [4-16,22].

In this paper, we have considered the boundary control problem for infinite order hyperbolic system and also for $(n \times n)$ infinite order hyperbolic systems involving constat time lags appearing both in the state equations and in the Neumann boundary conditions. We can also consider the boundary optimal control problem for $(n \times n)$ infinite order parabolic or hyperbolic systems with timevarying delays appearing in the state equations and in the Neumann or Dirichlet boundary conditions. We can also consider the boundary optimal control problem for $(n \times n)$ infinite order hyperbolic systems with timevarying delays appearing in the integral form with $h \in(a, b)$ or $h \in(0, b)$ both in the state equations and in the Neumann or Dirichlet boundary conditions.

Also it is evident that by modifying:

- The boundary conditions, (Dirichlet, Neumann, mixed, etc.);

- The nature of the control (distributed, boundary, etc.);

- The nature of the observation (distributed, boundary, etc.);

- The initial differential system;

- The time delays (constant time delays, time-varying delays, multiple time-varying delays, time delays given in the integral form, etc.);

- The number of variables (finite number of variables, infinite number of variables systems, etc.);

- The type of equation (elliptic, parabolic, hyperbolic, etc.);

- The order of equation (second order, Schrödinger, infinite order, etc.);

- The type of control (optimal control problem, timeoptimal control problem, etc.), an infinity of variations on the above problem are possible to study with the help of [21] and Dubovitskii-Milyutin formalisms [23-32]. Those problems need further investigations and form tasks for future research. These ideas mentioned above will be developed in forthcoming papers.

\section{REFERENCES}

[1] P. K. C. Wang, "Optimal Control of Parabolic Systems with Boundary Conditions Involving Time Delays," SIAM Journal on Control, Vol. 13, No. 2, 1975, pp. 274-293. doi: $10.1137 / 0313016$

[2] G. Knowles, "Time-Optimal Control of Parabolic Sys- tems with Boundary Conditions Involving Time Delays," Journal of Optimization Theory and Applications, Vol. 25, No. 4, 1978, pp. 563-574. doi:10.1007/BF00933521

[3] K. H. Wong, "Optimal Control Computation for Parabolic Systems with Boundary Conditions Involving Time Delays," Journal of Optimization Theory and Applications, Vol. 53, No. 3, 1987, pp. 475-507. doi:10.1007/BF00938951

[4] A. Kowalewski, "Optimal Control of Hyperbolic System with Boundary Condition Involving a Time-Varying Lag," Proceedings of the IMACS/IFAC International Symposium on Modeling and Simulation of DPS, Hiroshima, 6-9 October 1987, pp. 462-467.

[5] A. Kowalewski, "Boundary Control of Hyperbolic Systems with Boundary Condition Involving a Time Delay," Analysis and Optimization of Systems, Vol. 111, 1988, pp. 507-518. doi:10.1007/BFb0042240

[6] A. Kowalewski, "Optimal Control of Distributed Hyperbolic System with Boundary Condition Involving a Time Lag," Automatic Remote Control, XXXIII, 1988, pp. 537545.

[7] A. Kowalewski, "Optimal Control of Distributed Parabolic System Involving Time Lags," IMA Journal of Mathematical Control and Information, Vol. 7, No. 4, 1990, pp. 375-393. doi:10.1093/imamci/7.4.375

[8] A. Kowalewski, "Optimal Control of Parabolic Systems with Time-Varying Lags," IMA Journal of Mathematical Control and Information, Vol. 10, No. 2, 1993, pp. 113129. doi:10.1093/imamci/10.2.113

[9] A. Kowalewski, "Optimal Control of a Distributed Parabolic Systems with Multiple Time-Varying Lags," International Journal of Control, Vol. 69, No. 3, 1998, pp. 361-381. doi:10.1080/002071798222712

[10] A. Kowalewski, "Optimization of Parabolic Systems with Deviating Arguments," International Journal of Control, Vol. 72, No. 11, 1999, pp. 947-959. doi:10.1080/002071799220498

[11] A. Kowalewski and J. Duda, "On Some Optimal Control Problem for a Parabolic System with Boundary Condition Involving a Time-Varying Lag," IMA Journal of Mathematical Control and Information, Vol. 9, No. 2, 1992, pp. 131-146. doi:10.1093/imamci/9.2.131

[12] A. Kowalewski and J. Duda, "An Optimization Problem Time Lag Distributed Parabolic Systems," IMA Journal of Mathematical Control and Information, Vol. 21, No. 1, 2004, pp. 15-31. doi:10.1093/imamci/21.1.15

[13] W. Kotarski, H. A. El-Saify and G. M. Bahaa, "Optimal Control of Parabolic Equation with an Infinite Number of Variables for Non-Standard Functional and Time Delay," IMA Journal of Mathematical Control and Information, Vol. 19, No. 4, 2002, pp. 461-476. doi:10.1093/imamci/19.4.461

[14] W. Kotarski and G. M. Bahaa, "Optimality Conditions for Infinite Order Hyperbolic Problem with Non-Standard Functional and Time Delay," Journal of Information \& Optimization Sciences, Vol. 28, No. 6, 2007, pp. 315-334.

[15] H. A. El-Saify, "Optimal Control for $n \times n$ Parabolic System Involving Time Lag," IMA Journal of Mathe- 
matical Control and Information, Vol. 22, No. 3, 2005, pp. 240-250. doi:10.1093/imamci/dni011

[16] H. A. El-Saify, "Optimal Boundary Control Problem for $n$ $\times n$ Infinite Order Parabolic Lag System," IMA Journal of Mathematical Control and Information, Vol. 23, No. 4, 2006, pp. 433-445. doi:10.1093/imamci/dni065

[17] J. A. Dubinskii, "Sobolev Spaces of Infinite Order and the Behavior of Solution of Some Boundary Value Problems with Unbounded Increase of the Order of the Equation," Mathematics of the USSR-Sbornik, Vol. 27, No. 2, 1975, p. 143. doi:10.1070/SM1975v027n02ABEH002506

[18] J. A. Dubinskii, "Non-Triviality of Sobolev Spaces of Infinite Order for a Full Euclidean Space and a Torus," Mathematics of the USSR-Sbornik, Vol. 100, 1976, pp. 436-446.

[19] J. A. Dubinskii, "Sobolev Spaces of Infinite Order and Differential Equations," Springer-Verlag, New York, 1986.

[20] J. L. Lions and E. Magenes, "Non-Homogeneous Boundary Value Problem and Applications," Springer-Verlag, New York, 1972.

[21] J. L. Lions, "Optimal Control of Systems Governed by Partial Differential Equations," Springer-Verlag, New York, 1971.

[22] H. A. El-Saify and G. M. Bahaa, "Optimal Control for $n$ $\times n$ Hyperbolic Systems Involving Operators of Infinite Order," Mathematica Slovaca, Vol. 52, No. 4, 2002, pp. 409-424.

[23] G. M. Bahaa, "Quadratic Pareto Optimal Control of Parabolic Equation with State-Control Constraints and Infinite Number of Variables," IMA Journal of Mathematical Control and Information, Vol. 20, No. 2, 2003, pp. 167-178. doi:10.1093/imamci/20.2.167

[24] G. M. Bahaa, "Time-Optimal Control Problem for Infinite Order Parabolic Equation with Control Constraints," Differential Equations and Control Processes. The Electronic Journal, Vol. 4, 2005, pp. 64-81. http://www.neva.ru/journal

[25] G. M. Bahaa, "Optimal Control for Cooperative Parabolic
Systems Governed by Schrödinger Operator with Control Constraints," IMA Journal of Mathematical Control and Information, Vol. 24, No. 1, 2007, pp. 1-12. doi:10.1093/imamci/dn1001

[26] G. M. Bahaa, "Quadratic Pareto Optimal Control for Boundary Infinite Order Parabolic Equation with StateControl Constraints," AMO-Advanced Modeling and Optimization, Vol. 9, 2007, pp. 37-51.

[27] G. M. Bahaa, "Optimal Control Problems of Parabolic Equations with an Infinite Number of Variables and with Equality Constraints," IMA Journal of Mathematical Control and Information, Vol. 25, No. 1, 2008, pp. 37-48. doi:10.1093/imamci/dnm002

[28] G. M. Bahaa and W. Kotarski, "Optimality Conditions for $n \times n$ Infinite Order Parabolic Coupled Systems with Control Constraints and General Performance Index," IMA Journal of Mathematical Control and Information, Vol. 25, No. 1, 2008, pp. 49-57. doi:10.1093/imamci/dnm003

[29] W. Kotarski and G. M. Bahaa, "Optimal Control Problem for Infinite Order Hyperbolic System with Mixed Control-State Constraints," European Journal of Control, Vol. 11, No. 2, 2005, pp. 150-156. doi:10.3166/ejc.11.150-156

[30] W. Kotarski, H. A. El-Saify and G. M. Bahaa, "Optimal Control Problem for a Hyperbolic System with Mixed Control-State Constraints Involving Operator of Infinite Order," International Journal of Pure and Applied Mathematics, Vol. 1, No. 3, 2002, pp. 239-252.

[31] A. Kowalewski, "Time-Optimal Control of Infinite Order Hyperbolic Systems with Time Delays," International Journal of Applied Mathematics and Computer Science, Vol. 19, No. 4, 2009, pp. 597-608. doi:10.2478/v10006-009-0047-x

[32] A. Kowalewski and A. Krakowiak, "Time-Optimal Boundary Control of Infinite Order Parabolic System with Time Lags," International Journal of Applied Mathematics and Computer Science, Vol. 18, No. 2, 2008, pp. 189-198. doi:10.2478/v10006-008-0017-8 